\title{
Effect of Coronary Stenosis Severity on Variability of Quantitative Arteriography, and Implications for Interventional Trials
}

\author{
G. B. John Mancini, MD, Paula R. Williamson, BS, and Scott F. DeBoe, BS
}

$\mathbf{Q}$ uantitative coronary arteriography is now routinely used in studies assessing arterial remodelling in response to interventions such as lipid lowering and percutaneous transluminal coronary angioplasty. Although this methodology provides both relative and absolute measurements that can be evaluated statistically as continuous variables, it is, however, often desirable or necessary to establish categorical responses to the interventions based on the variability inherent in the quantitative methods (i.e., it is often necessary to state whether individual patients, as opposed to entire groups, have "restenosed," "progressed," "regressed," and so forth, and these categorical designations are based on critical limits of variability). ${ }^{1,2}$ These limits are generally based on an analysis of a rather diverse range of lesion severity, and the question arises as to whether the variability of measuring changes in lesion or segment morphology is affected by the initial severity of the lesion being studied. It is con-

From the Department of Internal Medicine, Division of Cardiology, University of Michigan and Veterans Administration Medical Centers (111A), 2215 Fuller Road, Ann Arbor, Michigan 48105. Manuscript received September 23, 1991; revised manuscript received November 22, 1991, and accepted November 30. ceivable that variability may be greater at 1 end than at the other end of the spectrum of lesion severity. If this is true, then the use of 1 critical value to designate whether a subject has responded in a certain way would be inappropriate, and this designation would be better achieved by using criteria that more directly reflect the measurement variability of lesions of a specified, initial severity. This report examines whether the variability of measuring morphologic parameters of stenoses is affected by the baseline severity of the lesion.

Records of digitally archived cineangiographic image frames (1 view) of narrowings previously acquired were retrospectively reviewed to establish a data set of 50 lesions distributed evenly among 5 categories of percent diameter stenosis severity ( 0 to 20,21 to 40,41 to 60,61 to 80 , and 81 to $99 \%$ ). One technician retrieved these images and reanalyzed them without knowledge of the originally determined stenosis severity. These images were then reanalyzed at an interval of $\geq 1$ week (mean 2 weeks) by the same technician without knowledge of the prior results. Each analysis consisted of an assessment of the minimum, maximum and mean diameter of the segment, as well as percent diameter stenosis. ${ }^{3}$ Within

\begin{tabular}{|c|c|c|c|c|c|c|}
\hline & \multicolumn{6}{|c|}{ Initial Severity } \\
\hline & 0 to $20 \%$ & 21 to $40 \%$ & 41 to $60 \%$ & 61 to $80 \%$ & 81 to $99 \%$ & All \\
\hline \multicolumn{7}{|l|}{ Minimum diameter $(\mathrm{mm})$} \\
\hline Mean & $2.4 \pm 0.7$ & $1.9 \pm 0.4$ & $1.6 \pm 0.3$ & $1.3 \pm 0.4$ & $0.4 \pm 0.3$ & $1.5 \pm 0.8$ \\
\hline Range & $1.3-4.0$ & $1.2-2.5$ & $1.2-2.4$ & $0.7-1.9$ & $0.1-1.1$ & $0.1-4.0$ \\
\hline Accuracy & 0.03 & 0.01 & 0.00 & -0.02 & -0.01 & 0.00 \\
\hline Precision & 0.12 & 0.13 & 0.13 & 0.05 & 0.14 & 0.11 \\
\hline Mean absolute difference & 0.09 & 0.09 & 0.11 & 0.04 & 0.10 & 0.10 \\
\hline rvalue & 0.99 & 0.94 & 0.95 & 0.99 & 0.89 & 0.99 \\
\hline \multicolumn{7}{|l|}{ Maximum diameter $(\mathrm{mm})$} \\
\hline Mean & $3.1 \pm 0.7$ & $3.2 \pm 0.6$ & $3.5 \pm 0.7$ & $4.0 \pm 1.1$ & $3.1 \pm 1.3$ & $3.4 \pm 1.0$ \\
\hline Range & $1.8-4.6$ & $2.1-4.2$ & $2.2-4.5$ & $2.7-6.2$ & $1.5-6.1$ & $1.5-6.2$ \\
\hline Accuracy & 0.13 & -0.04 & 0.00 & -0.20 & -0.03 & -0.03 \\
\hline Precision & 0.20 & 0.31 & 0.22 & 0.21 & 0.24 & 0.25 \\
\hline Mean absolute difference & 0.18 & 0.23 & 0.17 & 0.24 & 0.17 & 0.20 \\
\hline rvalue & 0.97 & 0.91 & 0.96 & 0.98 & 0.98 & 0.97 \\
\hline \multicolumn{7}{|l|}{ Mean diameter $(\mathrm{mm})$} \\
\hline Mean & $2.7 \pm 0.7$ & $2.4 \pm 0.4$ & $2.4 \pm 0.4$ & $2.7 \pm 0.8$ & $1.9 \pm 0.9$ & $2.4 \pm 0.7$ \\
\hline Range & $1.6-4.4$ & $1.6-3.3$ & $1.7-3.3$ & $1.6-4.5$ & $0.9-4.3$ & $0.9-4.5$ \\
\hline Accuracy & 0.04 & -0.01 & 0.07 & -0.10 & 0.00 & 0.00 \\
\hline Precision & 0.11 & 0.16 & 0.13 & 0.11 & 0.20 & 0.15 \\
\hline Mean absolute difference & 0.08 & 0.13 & 0.12 & 0.11 & 0.15 & 0.12 \\
\hline rvalue & 0.99 & 0.94 & 0.97 & 0.99 & 0.98 & 0.98 \\
\hline \multicolumn{7}{|l|}{ Percent diameter stenosis } \\
\hline Mean & $15 \pm 4$ & $34 \pm 5$ & $51 \pm 6$ & $66 \pm 4$ & $85 \pm 4$ & $50 \pm 25$ \\
\hline Range & $6-20$ & $20-39$ & $43-59$ & $61-73$ & $81-93$ & $6-93$ \\
\hline Accuracy & -1.7 & -2.0 & -0.5 & -0.9 & 0.1 & -1.0 \\
\hline Precision & 2.1 & 4.4 & 2.8 & 1.7 & 2.8 & 2.9 \\
\hline Mean absolute difference & 2.2 & $4.0^{*}$ & 2.2 & $1.4^{*}$ & 2.3 & 2.4 \\
\hline rvalue & 0.86 & 0.65 & 0.89 & 0.93 & 0.74 & 0.99 \\
\hline
\end{tabular}


each severity subgroup and for each anatomic measurement, correlation coefficient ( $r$ value), accuracy (mean differences between the 2 measurements, retaining the direction of the difference), precision (standard deviation of mean differences) and mean absolute difference (mean absolute difference between measurements; i.e., direction of the difference was ignored) were calculated. For these analyses, the first set of measurements was considered the standard by which the second set was compared. In contrast to other reports, ${ }^{2}$ this study reflects only the variability that can be ascribed to baseline lesion severity and does not include the increased variability that can be expected when frame reselection and redigitization are involved.

Correlation coefficients were assessed using a Bonferroni-adjusted Fisher's Z transformation. Repeated measures analysis of variance was used for assessing accuracy and mean absolute difference values. In the case of mean absolute differences, a Newman-Keuls simultaneous comparisons test was used to determine which subgroups were significantly different. Precision was assessed using a Hartley test for heterogeneous variances. Results were considered significant at $p<0.05$.

Table I shows the study results and includes an assessment of the overall variability in the entire cohort. There were no significant differences among any of the absolute measures of stenosis severity. The mean absolute difference of the percent diameter stenosis parameter was higher in the 21 to $40 \%$ severity group than in the 61 to $80 \%$ one $(4.0 \pm 2.4 \%$ vs $1.4 \pm 1.1 \%$; $p<0.01)$. These 2 values were not significantly different from any of the other mean absolute difference values (range 2.2 to $2.4 \%$ ) in the other subgroups.
This study demonstrates that the variability of absolute stenosis dimensions is not significantly affected by the initial severity of the stenosis being measured. The percent diameter stenosis measurement showed a minor tendency to be more variable in the modest ( 21 to $40 \%$ ) than in a more severe (61 to $80 \%$ ) group. This was manifest as a statistically significant difference in the mean absolute difference in repeated measures and was supported by a lower (although not significantly) correlation coefficient. Therefore, this study suggests that 1 categorical criterion can be used to assess responses to therapy and intervention, which are not affected by the initial severity of the lesion when absolute measurements are used. For most purposes, the same probably applies to measures of percent diameter stenosis. However, it may be prudent for investigators interested in following a particular subset of stenoses (e.g., "new lesion formation") to create quantitative limits for categoric determinations based specifically on the range of severity of the type of lesion to be investigated. ${ }^{4}$

1. Reiber JHC, Serruys PW, Kooijiman CJ, Wijns W, Slager CJ, Gerbrands JJ Schuurbiers JCH, den Boer A, Hugenholtz PG. Assessment of short-, mediumand long-term variations in arterial dimensions from computer-assisted quantitation of coronary cineangiograms. Circulation 1985;71:280-288.

2. deCesare NB, Williamson PR, Moore NB, DeBoe SF, Mancini GBJ. Establishing comprehensive, quantitative criteria for detection of restenosis and remodelling after percutaneous transluminal coronary angioplasty. Am J Cardio 1992;69:in press.

3. Mancini GBJ, Simon SB, McGillem MJ, LeFree MT, Friedman HZ, Voge RA. Automated quantitative coronary arteriography: morphologic and physiolog. ic validation in vivo of a rapid digital angiographic method. Circulation 1987;75:452-460.

4. Lichtlen PR, Hugenholtz PG, Rafflenbeul W, Hecker H, Jost S, Deckers JW Retardation of the angiographic progression of coronary artery disease in man by the calcium channel blocker nifedipine-results of the international nifedipine trial on antiatherosclerotic therapy (INTACT). Lancet 1990;335:1109-1113.

\title{
Coronary Artery Disease and Factor VII Hyperactivity in Elderly Japanese
}

\author{
Kazuomi Kario, MD, Takefumi Matsuo, MD
}

H igh levels of factor VII (FVII) coagulant activity (FVIIc) have been associated with an increased risk of coronary artery disease and cardiac death, and are thought to be of greater significance than cholesterol in the first 5 years after screening. ${ }^{1}$ These studies have mainly been performed in Western countries and the subjects were generally adults aged $<65$ years. Japan is one of the countries where the incidence of coronary artery disease is low, ${ }^{2}$ and we have recently reported on FVII hyperactivity in the elderly Japanese. ${ }^{3}$ This time, to study the relation between coronary artery disease and FVII in elderly Japanese persons, we performed electrocardiography and measured FVIIc and FVII antigen (FVIIag) levels as well as blood lipid fractions.

From the Department of Internal Medicine, Hyogo Prefectural Awaj Hospital and Awaji-Hokutan Public Clinic, Hyogo, Japan. This study was supported in part by a grant from the Foundation for the Development of the Community, Tochigi, Japan. Dr. Kario's address is: 480-2, Ikuha, Hokutan-chyo, Tsuna-gun, Hyogo-ken, 656-16, Japan. Manuscript received September 9, 1991; revised manuscript received and accepted November 14, 1991.
A total of 317 elderly Japanese subjects (>60 years old), who were residents of a local home for the aged, were studied. Fifty-one of the 127 subjects (40\%) with hypertension and 27 of the 37 subjects $(73 \%)$ with diabetes mellitus were receiving medication. Eighteen subjects received antihyperlipidemic agents. An electrocardiogram was recorded in all subjects. Subjects who did not have hypertension, diabetes mellitus, peripheral vascular disease, hyperlipidemia, a history of stroke, or an ischemic electrocardiographic tracing were classified as the healthy elderly group ( 82 men, mean age $77 \pm 9$ years; 62 women, mean age $80 \pm 7$ years). Blood samples were collected after a 12-hour fast. FVIIc was assayed by an automated l-stage method (Coag-stat, Kyoto Daiichi Pharmacological Co.), using human FVII-deficient plasma (Dade, France) and rabbit calcified thromboplastin reagent (Thromboplastin $C$, Dade, France). FVIIag was assayed by an ELISA (Diagnostica Stago, France). In brief, diluted plasma samples or calibulators (normal plasma pool of 20 nonsmoking men aged $<30$ years) were added to the microwells that had been coated 